Supporting Information

\title{
Oxygen Electroreduction Catalyzed by Palladium Nanoparticles Supported on Nitrogen-Doped Graphene Quantum Dots: Impacts of Nitrogen Dopants
}

\author{
Christopher P. Deming ${ }^{\dagger}$, Rene Mercado ${ }^{\dagger}$, Jia En Lü ${ }^{\dagger}$, Vamsi Gadiraju ${ }^{\ddagger}$, Mohammad Khan ${ }^{\dagger}$ and Shaowei \\ Chen $^{t, *}$ \\ ${ }^{\dagger}$ Department of Chemistry and Biochemistry, University of California, 1156 High Street, Santa Cruz, \\ California 95064. *E-mail: shaowei@ucsc.edu \\ ‡ The Harker School, 4300 Bucknell Road, San Jose, California 95130
}

(11 figures and 7 pages)

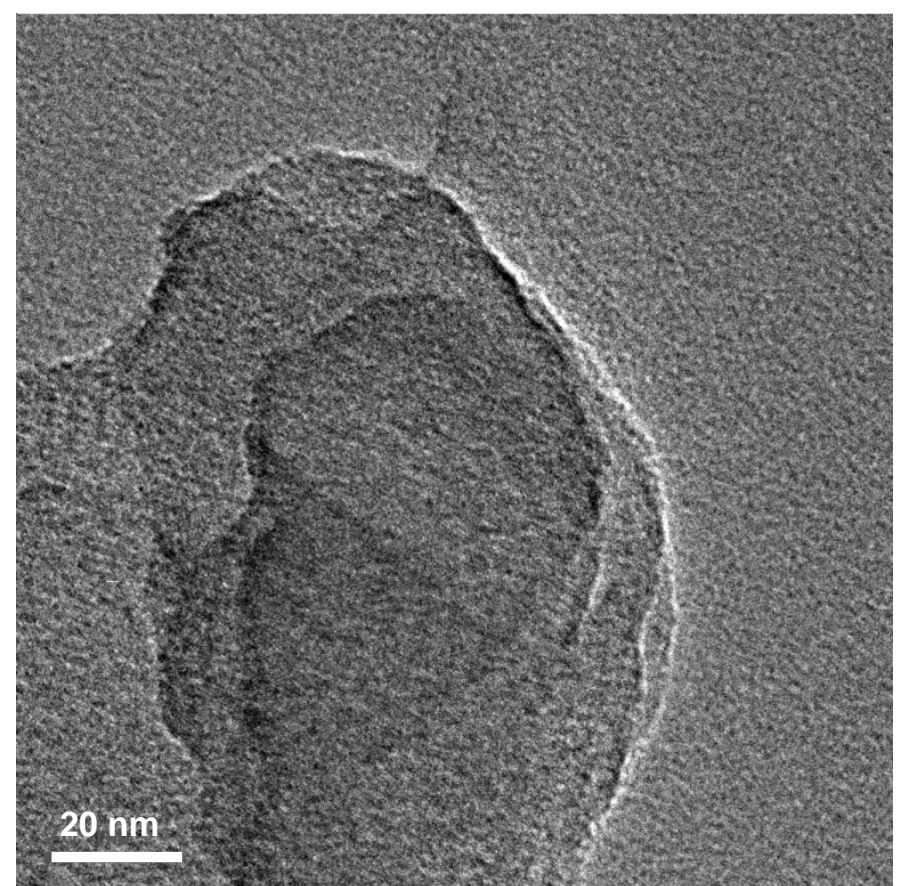

Figure S1. Representative TEM image of NGQD synthesized by thermal treatment of citric acid and urea. Scale bar $20 \mathrm{~nm}$. 

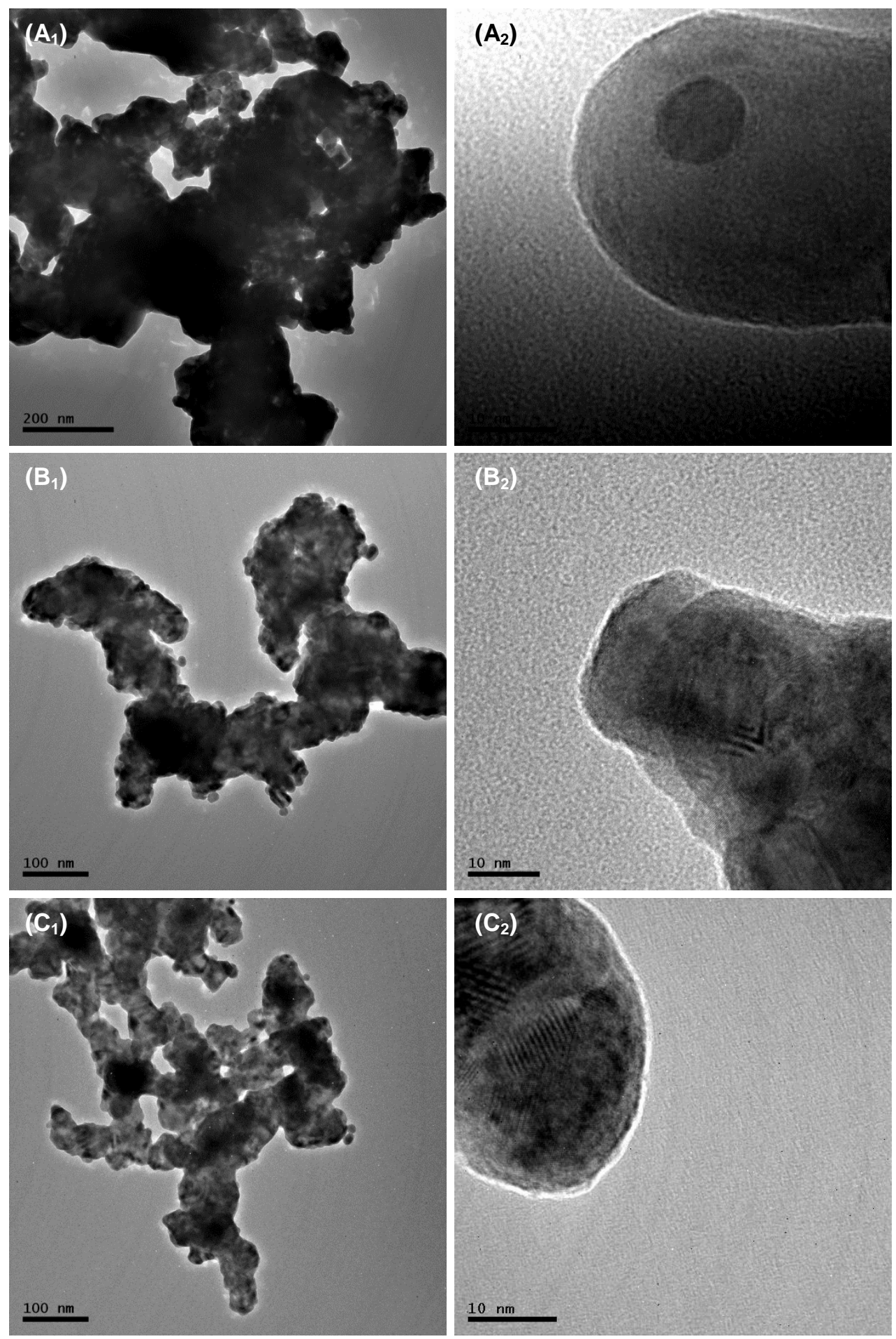

Figure S2. Representative TEM images of $\left(A_{1}, A_{2}\right)$ PdGQD-1, $\left(B_{1}, B_{2}\right)$ PdGQD-4, and $\left(C_{1}, C_{2}\right)$ PdGQD-8 nanocomposites. Scale bars are $200 \mathrm{~nm}\left(A_{1}\right), 100 \mathrm{~nm}$ in $\left(B_{1}\right)$ and $\left(C_{1}\right)$, and $10 \mathrm{~nm}$ in $\left(A_{2}\right),\left(B_{2}\right)$ and $\left(C_{2}\right)$. 

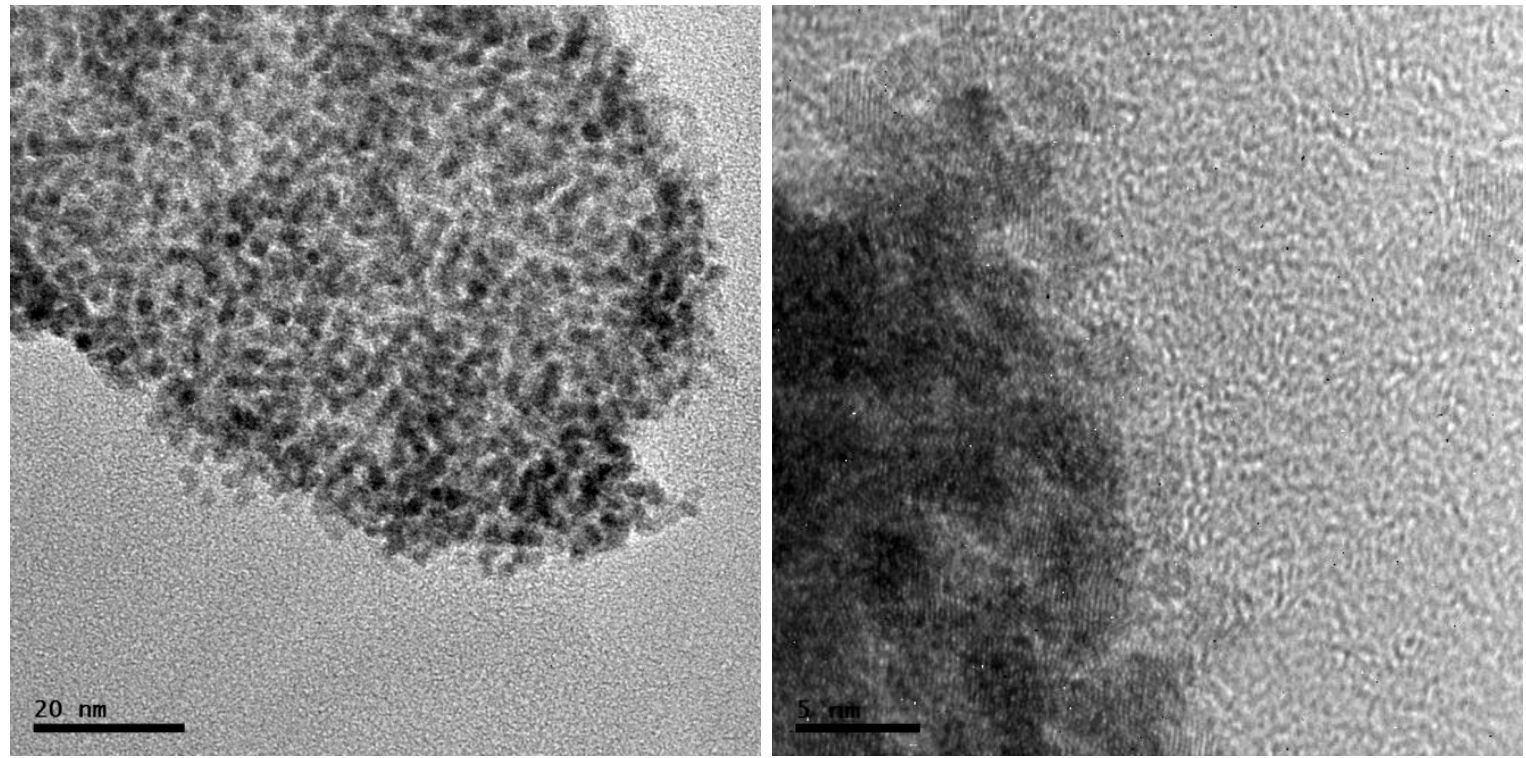

Figure S3. Representative TEM images of RuNGQD nanocomposites. Scale bars are $20 \mathrm{~nm}$ in the left and $5 \mathrm{~nm}$ in the right. It can be seen that RuNGQD composites exhibited similar morphological characteristics to the PdNGQD samples with small subunits clustered in larger superstructures larger than $100 \mathrm{~nm}$ (left panel). Additionally, HRTEM images (right panel) showed well defined lattice fringes consistent with the $100(0.234 \mathrm{~nm})$ and $002(0.214 \mathrm{~nm})$ crystalline planes of Ru. XPS measurements also displayed peaks centered at $398.5 \mathrm{eV}$ and $400.0 \mathrm{eV}$, indicating the formation of pyridinic and pyrrolic nitrogen centers at the concentration of $24.5 \%$ and $75.5 \%$, respectively. Again, there is no signal from quaternary nitrogen centers. Raman measurements (details in Figure S7) showed the characteristic D and $G$ bands, along with the $I_{D} / I_{G}$ ratio, that were consistent with those of the PdNGQD-8 sample.

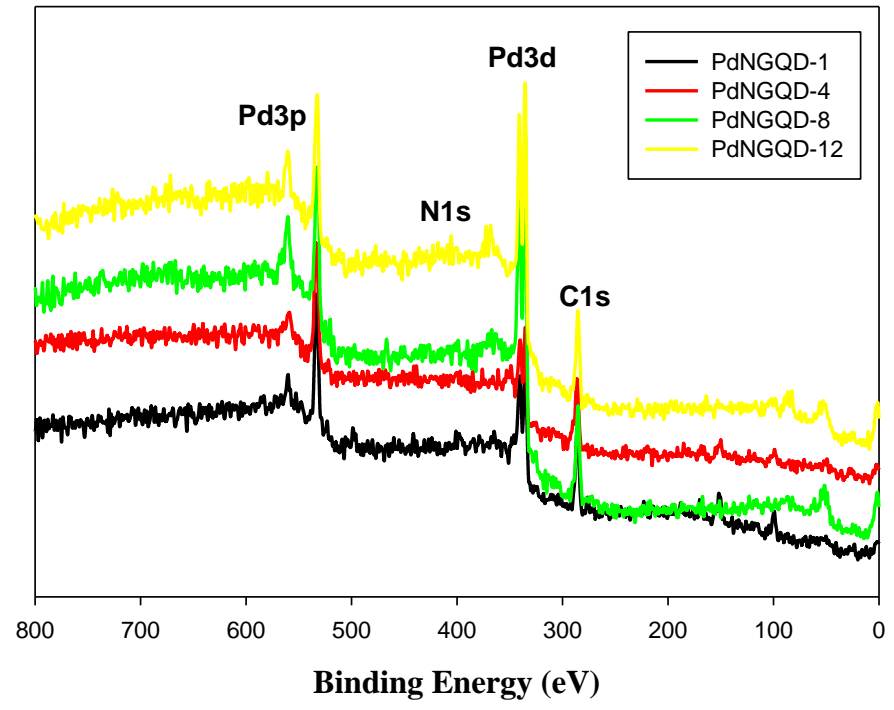

Figure S4. XPS survey spectra of the series of PdNGQD nanocomposites. 


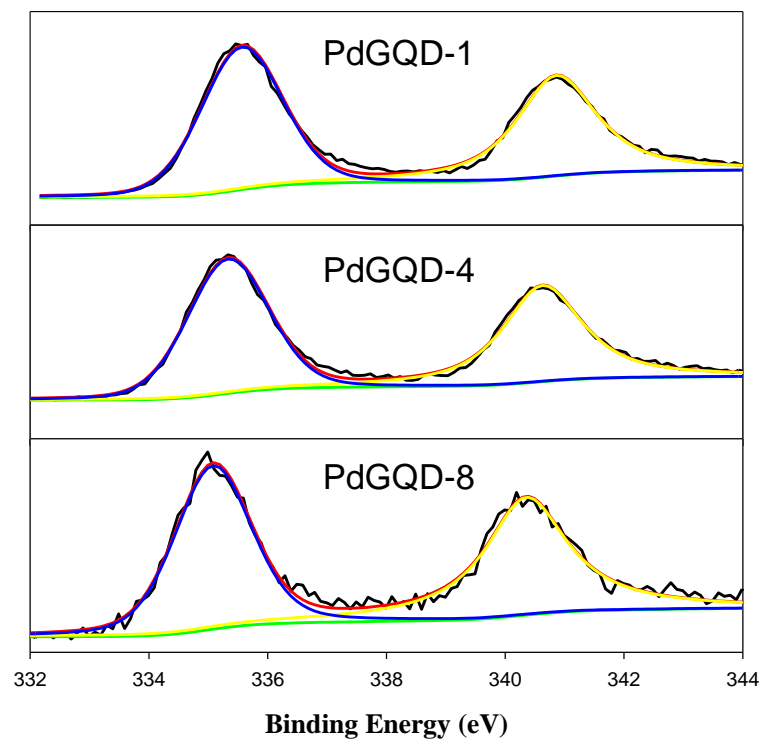

Figure S5. High-resolution XPS spectra of Pd3d electrons of the series of PdGQD nanocomposites. Black curves are experimental data and colored curves are deconvolution fits.

\begin{tabular}{|c|c|c|}
\hline & Pd3d5/2 (eV) & Pd3d3/2 (eV) \\
\hline PdGQD-1 & 335.6 & 340.8 \\
\hline PdGQD-4 & 335.4 & 340.6 \\
\hline PdGQD-8 & 335.1 & 340.5 \\
\hline
\end{tabular}

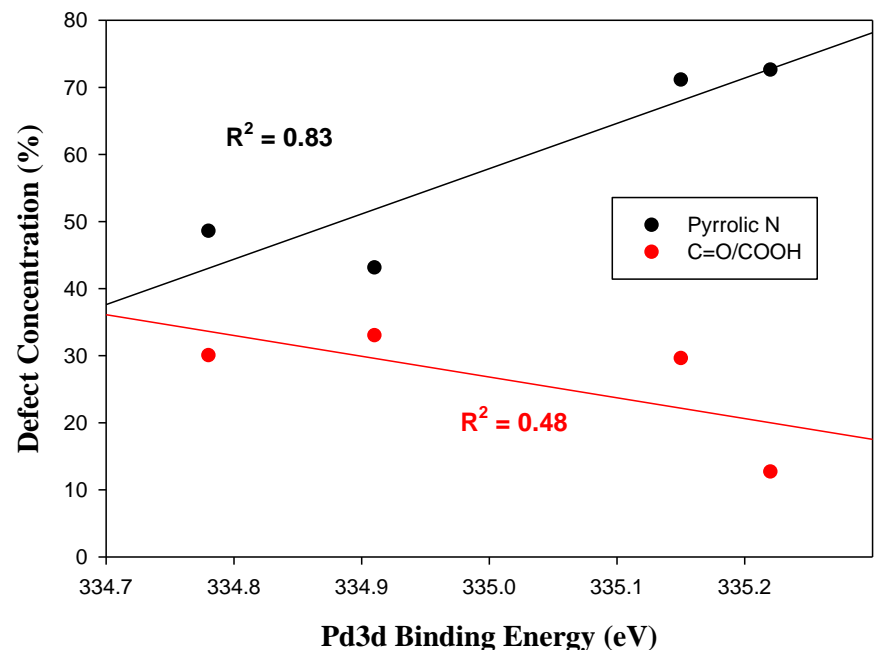

Figure S6. Correlation between $\mathrm{Pd} 3 \mathrm{~d}_{5 / 2}$ binding energy and the concentrations of pyrrolic $\mathrm{N}$ and $\mathrm{C}=\mathrm{O} / \mathrm{COOH}$ defects in PdNGQD. Symbols are experimental data and lines are linear regressions (the corresponding $\mathrm{R}^{2}$ coefficients are also listed in the figure). 


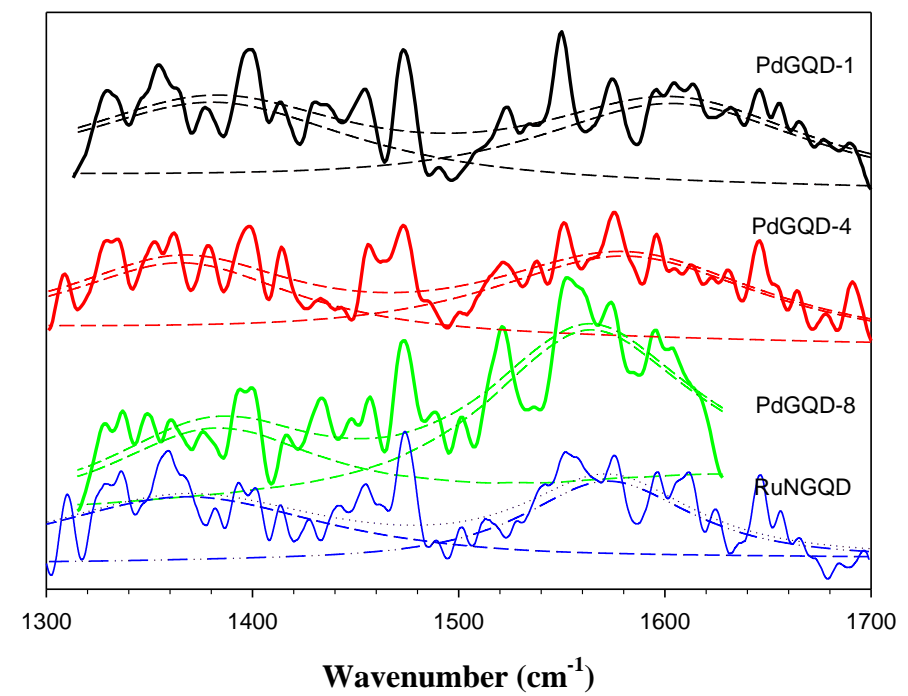

Figure S7. Raman spectra of PdGQD and RuNGQD nanocomposites. Solid curves are experimental data and dashed curves are deconvolution fits.

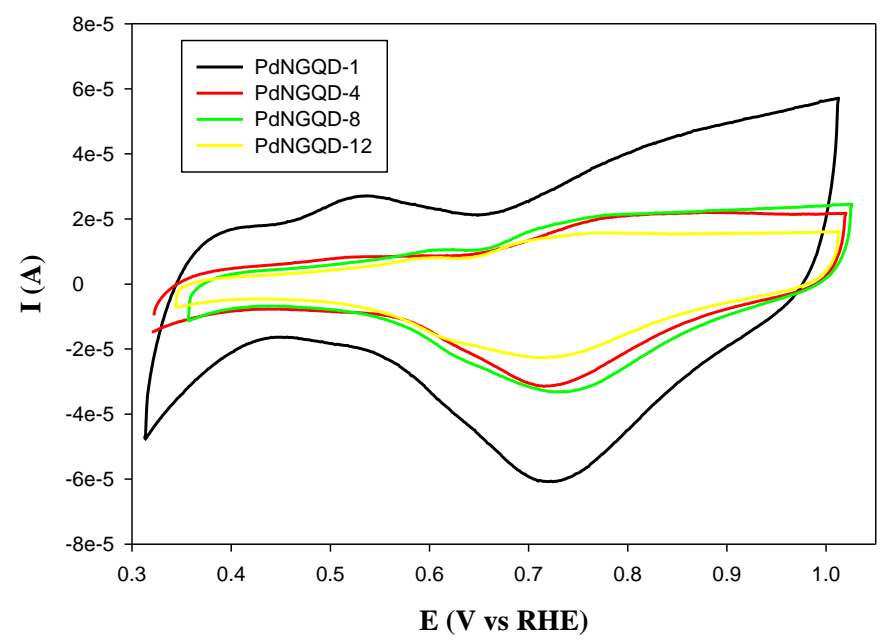

Figure S8. Cyclic voltammogram of PdNGQD nanocomposites in a nitrogen-saturated $0.1 \mathrm{M} \mathrm{KOH}$ solution. Potential scan rate $50 \mathrm{mV} / \mathrm{s}$. The palladium loading is $5.9 \mu \mathrm{g}$ for PdNGQD-1, $7.2 \mu \mathrm{g}$ for PdNGQD-4, $5.7 \mu \mathrm{g}$ for PdNGQD-8, and 4.5 $\mu \mathrm{g}$ for PdNGQD-12. 

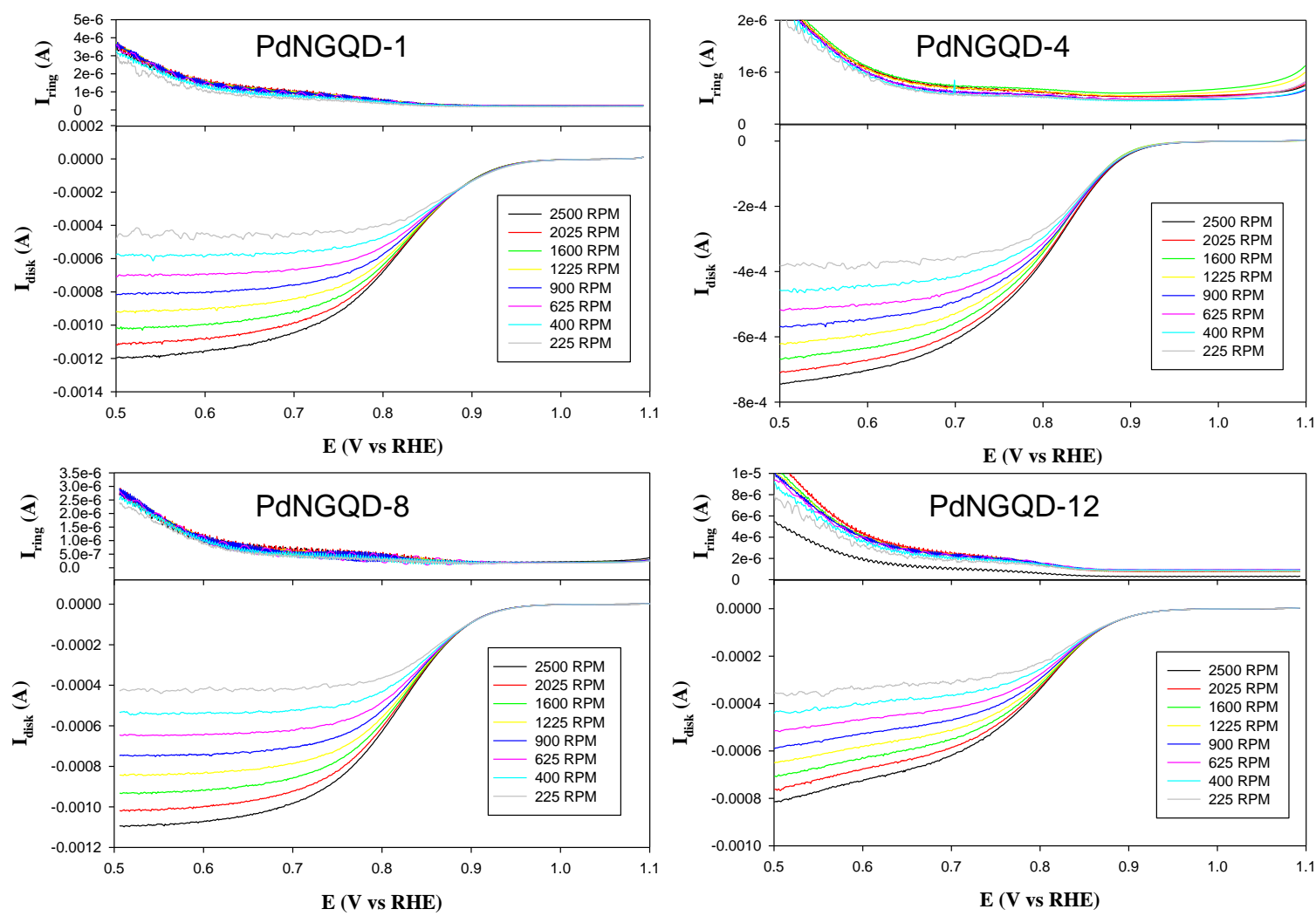

Figure S9. RRDE voltammograms of the series of PdNGQD nanocomposites in oxygen-saturated $0.1 \mathrm{M}$ $\mathrm{KOH}$ at various electrode rotation rates (which are specified in the figure legends). Other experimental conditions are the same as those in Figure 5. 

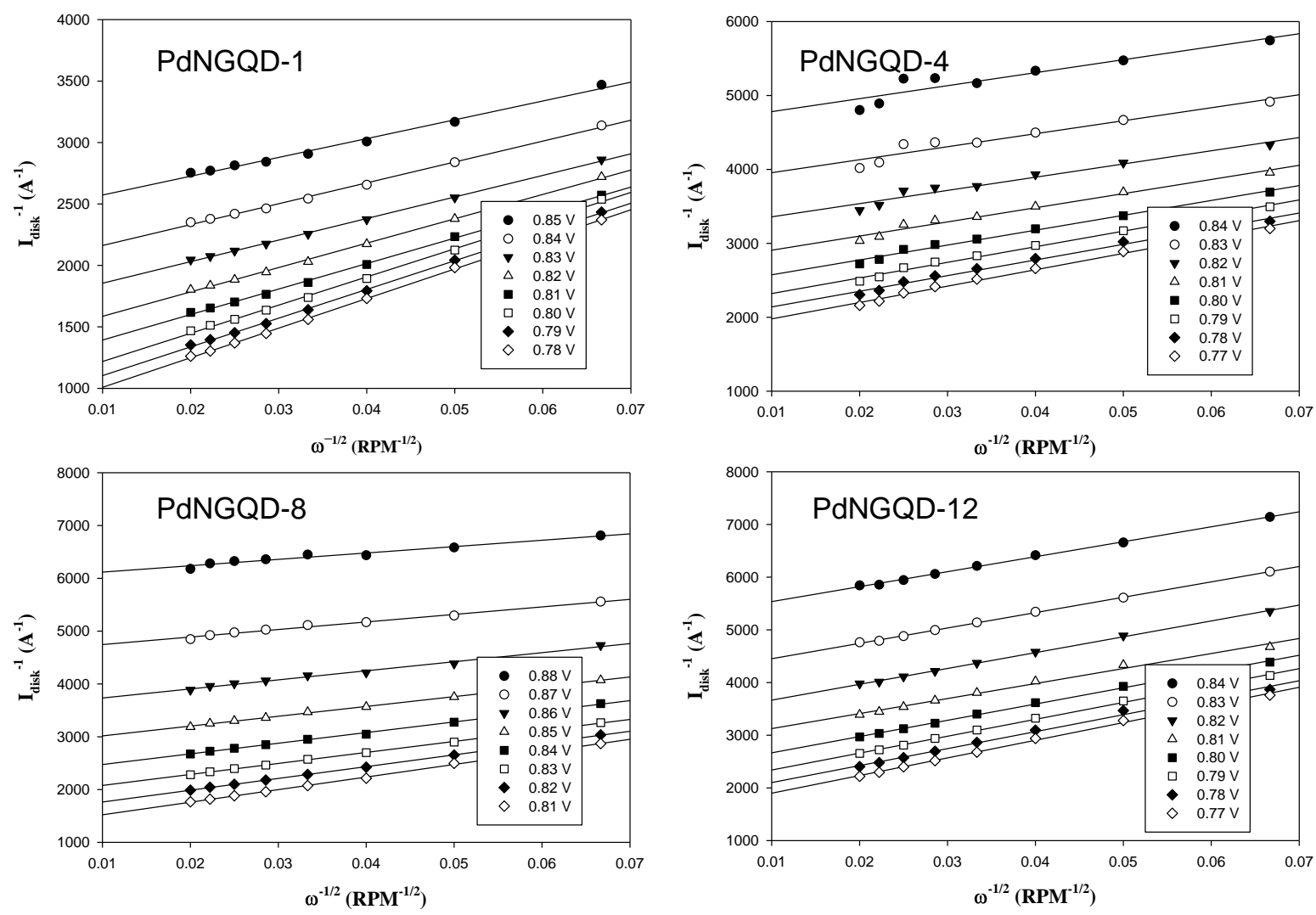

Figure S10. Koutecky-Levich plots of the series of PdNGQD nanocomposites in ORR. Symbols are experimental data obtained from Figure $\$ 9$ and lines are linear regressions.

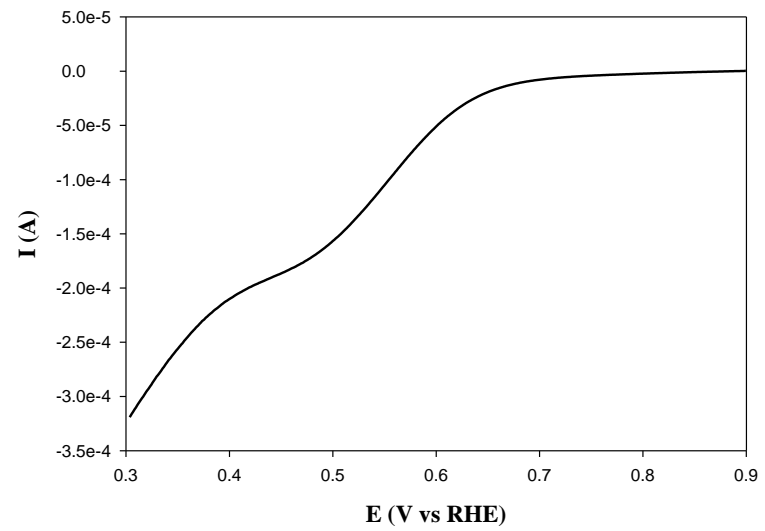

Figure S11. RDE voltammogram of RuNGQD in in oxygen-saturated $0.1 \mathrm{M} \mathrm{KOH}$ at the electrode rotation rate of 1600 RPM. Other experimental conditions are the same as those in Figure 7. Ruthenium metal loading was ca. $7 \mu \mathrm{g}$. 